\title{
LA PIEDRA DE LA LOCURA: INICIOS HISTÓRICOS DE LA SALUD MENTAL
}

\author{
Oswaldo Salaverry $1,2, a$
}

\section{RESUMEN}

Sobre la evidencia de la relativamente reciente preocupación de la salud pública por los problemas de salud mental, se revisa la evolución del concepto de enfermedad mental en la historia premoderna de occidente y las razones por las cuales se mantuvo fuera de la corriente principal de la actividad sanadora profesional. Asimismo, se explora las distinciones entre la visión naturalista y sobrenatural y su relación entre lo público y lo privado como explicación de su tardía incorporación como problema de Salud Pública.

Palabras clave: Salud mental; Historia de la medicina; Salud pública (fuente: DeCS BIREME).

\section{THE STONE OF MADNESS: STARTING POINTS OF THE HISTORY OF MENTAL HEALTH}

\section{ABSTRACT}

Addressing the relatively recent concern of public health in the issue of mental health problems, the evolution of the concept of mental illness in the occidental pre-modern history is revised, as well as the reasons why it remained outside the mainstream of the professional healing activity. The differences between the naturalist and the supernatural views are explored as well.

Key words: Mental health; History of medicine; Public health (source: MeSH NLM).

Quem deus vult perdere dementat Prius

(Los dioses privan de la razón a quienes quieren perder) ${ }^{(1)}$

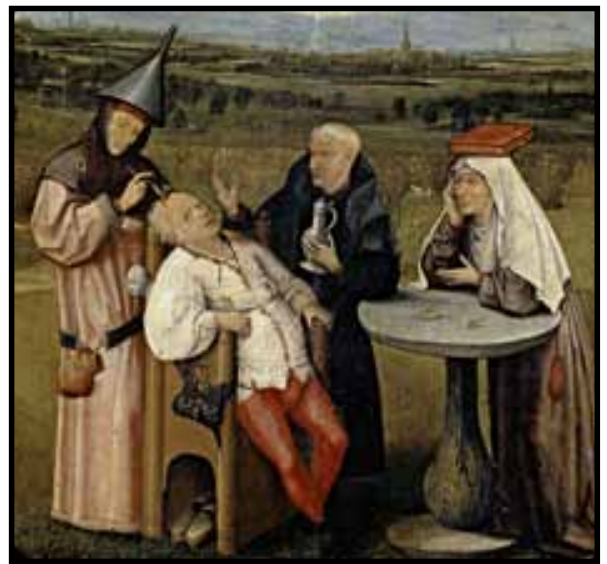

Figura 1. Extracción de la piedra de la locura. Jeroen Anthoniszoon van Aeken, conocido como El Bosco, pintada hacia 1475-1480. Óleo sobre tabla, $48 \mathrm{~cm} \times 35 \mathrm{~cm}$, conservado en el Museo del Prado, Madrid, España.

Centro Nacional de Salud Intercultural, Instituto Nacional de Salud. Lima, Perú.

2 Facultad de Medicina Humana, Universidad Nacional Mayor de San Marcos. Lima, Perú.

a Médico, doctor en Medicina

Recibido: 07-03-12 Aprobado: 07-03-12 


\section{SALUD PÚBLICA Y SALUD MENTAL}

En la agenda de la Salud Pública contemporánea, la salud mental ocupa un lugar destacado, en gran medida como resultado de diversas estimaciones que pronostican su notable deterioro global en un futuro inmediato. La tardía identificación de la salud mental como problema de Salud Pública responde a un complejo conjunto de interrelaciones entre lo que se percibe como del ámbito público y lo que pertenece al ámbito íntimo y doméstico. A su vez, esta percepción deriva de conceptos y distinciones sociales previas, como aquellos que definen lo sano y lo enfermo de acuerdo con circunstancias socioculturales concretas que evolucionan históricamente. Desde una perspectiva histórica, las enfermedades mentales se han encontrado constantemente en los límites entre las interpretaciones naturalistas y sobrenaturales de la enfermedad, lo que también ha contribuido a su tardía incorporación como problema de salud.

Abordar esta compleja trama de circunstancias nos remonta a los orígenes de la medicina occidental.

\section{LA LOCURA EN LA HISTORIA}

Los primeros informes en occidente sobre problemas de salud mental los encontramos en la literatura sagrada. En el libro de Daniel del antiguo testamento se relata la locura de Nabucodonosor, castigado por su soberbia con la locura, la pérdida de la razón; la que, por cierto, es interpretada como vivir y comer como los animales. Durante siete años Nabucodonosor permanece en ese estado hasta que por decisión divina se recupera.

El relato muestra la interpretación, generalizada en la antigüedad, sobre el origen sobrenatural de la enfermedad mental, compartida tanto por los legos como por los sanadores profesionales. Las culturas más complejas atribuían la locura a los dioses, las más primitivas a los demonios o fuerzas naturales, pero no son diferencias esenciales, derivan del pensamiento primitivo, aquel que dio lugar a las trepanaciones craneales, practicadas desde hace más de 5000 años, con evidencias en casi todo el mundo y que aparentemente tenían como una de sus indicaciones el dejar salir los demonios que provocaban las enfermedades mentales.

La interpretación naturalista de las enfermedades mentales se inicia en Grecia hacia el siglo $V$ a.C. al extenderse la teoría humoralista de la enfermedad a los problemas o manifestaciones mentales. El humoralismo como doctrina interpretaba la salud como el equilibrio de los cuatro fluidos o "humores" que conformaban el cuerpo humano, a saber: la bilis negra, la bilis amarilla, la flema o pituita y la sangre. Así, una fiebre o un problema digestivo se interpretaba como resultado de un particular desequilibrio en la composición humoral de los órganos afectados, ante el cual la labor del médico era restablecer el balance perdido. Una característica de la teoría humoral es que situaba topográficamente el desequilibrio, por lo tanto, en el caso de las enfermedades mentales debía ubicar el lugar preciso del desequilibrio que las causaba, y ese lugar era claramente el cerebro. Esta interpretación somatista de la enfermedad debió luchar contra la ampliamente difundida y hegemónica interpretación de la posesión demoníaca, la cual por cierto había tomado también en la cultura griega la forma más sofisticada de "entusiasmo", una posesión por los dioses o "inspiración" que llevaba a profetizar, hablar en lenguas o hacer extrañas contorsiones a quienes eran así poseídos.

En particular las convulsiones súbitas eran un atributo de augures o personajes elegidos por los dioses, por ello se le denominaba la "enfermedad sagrada". En el Corpus Hippocraticum, es decir el conjunto de escritos que la antigüedad atribuía a Hipócrates, se encuentra un breve tratado dedicado al tema (Sobre la enfermedad sagrada) el cual inicia con el siguiente enunciado: $A$ propósito de la llamada enfermedad sagrada, he aquí lo que ocurre: me parece que no es en modo alguno más divino ni más sagrado que las demás enfermedades, sino que tiene una causa natural. Pero los hombres creyeron que su causa era divina por ignorancia o por el carácter maravilloso de la dolencia, que no se parece en nada a otras enfermedades (2).

Cabe señalar, sin embargo, que existía otra explicación para las convulsiones específicamente femeninas: la curiosa concepción de que el útero es móvil y en sus traslados, cuando afectaba al hígado provocaba convulsiones ${ }^{(3)}$.

Galeno, que sistematizó la medicina griega en el siglo II d.C., no dedicó un tratado especial dentro de su abundante obra a la enfermedad mental, pero aceptaba, igual que los hipocráticos, su carácter natural. La tradición galénica clasificaba las enfermedades mentales en dos tipos: la manía y la melancolía. La primera se produciría por un exceso o plétora, bien sea del humor sangre o de la bilis amarilla, y se manifestaría con alucinaciones o delusiones, mientras que la melancolía se originaba en un exceso de la bilis negra y su principal manifestación seria la depresión. Esta clasificación se mantuvo durante el Imperio romano, quienes a su vez desarrollaron las primeras manifestaciones de la salud pública bajo la forma del cuidado de la salud de los 
ciudadanos (acueductos, cloacas, control de alimentos) e incluso un esbozo de lo que serán hospitales públicos, pero la enfermedad mental continuó siendo un tema de carácter intimo, atendido dentro del hogar, en modo alguno percibido como un tema de la res publica o asuntos que competen al estado.

La difusión del cristianismo, y su posterior oficialización a partir del decreto de Constantino en el 313 d.C., condujo a inevitables conflictos entre el carácter naturalista de la ciencia y medicina grecorromana y la visión cristiana que enfatizaba la omnipotencia y omnipresencia divina. Los médicos convertidos al cristianismo supeditaron sus convicciones profesionales a las religiosas y, por tanto, entendieron la enfermedad como un designio divino. Se adoptó como dogma cristiano la eterna lucha entre el bien y el mal por el alma humana, resurgiendo entre los médicos cristianos la interpretación de la locura como posesión demoníaca. Es en esta joven sociedad cristiana de finales del imperio que surgen los primeros hospitales administrados por las matronas cristianas, como expresión de la caridad y compasión con el doliente pero, en modo alguno, pretendiendo alterar los designios divinos; por lo tanto, no estaban destinados a curar a los enfermos sino a brindarles apoyo espiritual y también físico en tanto se definía la voluntad divina al respecto. Con esa concepción se entiende que no hubiera lugar en ellos para los enfermos mentales pues la enfermedad mental no era propiamente una dolencia, era una forma de posesión sobrenatural, cuando no demoníaca, que debería ser, en todo caso, tratada por el sacerdote. Por consiguiente, el cuidado de los enfermos mentales se mantuvo en el ámbito doméstico y alejado del ámbito de la Salud Pública.

Por otro lado, mientras en occidente se va configurando la sociedad medieval, en la península arábiga nace el Islam. Antes de Mohammad, los árabes carecían de una medicina racional y atribuían las enfermedades, incluyendo los trastornos mentales, a fuerzas sobrenaturales. Mohammad que brinda unidad religiosa y política al pueblo árabe también recoge conocimientos y prácticas tradicionales, los que reunidos conformarán la denominada "medicina del profeta" (4), en la que la enfermedad mental solo se menciona para indicar que los amuletos y talismanes son inefectivos para evitarla. La tradición popular que la atribuye a seres sobrenaturales se mantiene como en el caso estudiado por Dols ${ }^{(5)}$. quien refiere el caso de un loco (majnun) que escapa de la sección de los insanos (mamruirin) de un hospital (maristan o bimaristan) y en sus andanzas se proclama emir, llegando a convencer a muchos con su historia; finalmente, se descubre su locura (junum) causando graves confusiones y problemas, los que tienen como único castigo el volver al hospital. La benignidad de la sanción probablemente se sustente en la difundida creencia de que la locura era causada por "genios" (jinn) o demonios y, por tanto, no eran atribuibles al individuo.

En el Islam, a diferencia de occidente, se acepta a los enfermos mentales en los hospitales; esto se debe a que los árabes adoptan la medicina griega llevada por los intelectuales emigrantes que huyen de la intolerancia cristiana, convirtiéndola en el centro de su doctrina, al punto que se utiliza como criterio de calidad profesional la mayor o menor adherencia a los principios galénicos. Se entiende así su interpretación naturalista y que los hospitales tuvieran una sección destinada al tratamiento de los enfermos mentales, en los que el tratamiento continuó siendo con purgas y sangrías para eliminar los humores alterados. Pese a este tratamiento profesional de la salud mental, la concepción popular predominante mantuvo a la enfermedad mental como un asunto familiar y doméstico.

En occidente el Medioevo se caracteriza por un empobrecimiento teórico de la Medicina y su subordinación a la religión, lo que lleva a extremos como la glorificación de la enfermedad entendida como una mortificación de la materia. Jacopone da Todi, un poeta místico del siglo XIII en su poema De l'Infermità e Mali che Frate Jacopone demandava per Eccesso de Carità reclama para sí un cúmulo de enfermedades físicas, pero ninguna de carácter mental, pues su vinculación con el demonio se mantenía vigente ${ }^{(6)}$. La salud pública tiene escasos desarrollos vinculados todos ellos a la progresiva consolidación de los burgos y villas, que deben tomar medidas de alcance local para prevenir o combatir plagas, los hospitales desaparecen $y$, de algún modo, son sustituidos por los infirmarium, instalados en los monasterios. El loco o lunático (término ese último acuñado por la supuesta influencia de la luna en el origen de la enfermedad mental) vaga por las calles asimilándose en algunos casos al bufón. Es una visión complaciente y pintoresca pero que mantiene a las enfermedades mentales alejadas de las preocupaciones de la salud pública que, en cambio, se orientan al aislamiento de los leprosos. La peste negra o gran plaga que arrasa Europa entre 1346 y 1400 , produce cuadros colectivos de alteraciones conductuales pero esto no modifica la percepción popular.

Hacia el final de la Edad Media (1511) Erasmo de Rotterdam publica su conocido ensayo "Elogio de la locura" (Morias enkomion), que no es un tratado médico 
pero muestra la interpretación intelectual renacentista de la locura, que artificiosamente es considerada una diosa, hija de Pluto, el dios de la riqueza y la ninfa Hebe, ninfa de la juventud ${ }^{(7)}$. Su interpretación influenciará y mantendrá la convicción anecdótica de la enfermedad mental.

\section{LA INTERPRETACIÓN MECANICISTA DE LA ENFERMEDAD MENTAL}

El mecanicismo gana espacio en la interpretación médica a partir de las doctrinas cartesianas que dividen al hombre en un componente material y otro racional (res extensa y res cogitans). El dualismo así formulado prefigura el actual problema de mente y cuerpo y tiene influencia directa en el lugar que ocupan las enfermedades mentales en la ciencia. Hasta la propia denominación "enfermedad mental", en una interpretación literal, encerraría una contradicción para los cartesianos pues por definición el alma o espíritu (mente) se encuentra fuera del mundo material en donde ocurren las enfermedades. Se sigue de ese razonamiento que la locura necesariamente debe ser una enfermedad del cuerpo; pero en ese caso, el problema se traslada a encontrar una explicación sobre cual es la relación entre la mente y el cuerpo, entre la voluntad y las funciones corporales y como es que una enfermedad que tiene una sede material y corporal puede afectar el comportamiento regido por la razón, es decir la res cogitans.

Los médicos cartesianos intentan diversas respuestas. Uno de ellos fue Thomas Willis, el anatomista a quien debemos términos como "neurología" y cuyo epónimo recordamos en el "polígono de Willis". En su interpretación, el cerebro se nutría como todos los órganos de sangre, pero la que llegaba al cerebro pasaba por un proceso previo de filtración que la convertía en productos sutiles o "espíritus animales", que se constituían en intermediarios entre el cuerpo físico y la mente. En esa línea de pensamiento la locura y una de sus manifestaciones, las delusiones o falsas ideas, eran resultado de la acción caótica de esos espíritus en la mente. Esos mismos espíritus podían llegar a los músculos y explicaban entonces los extraños movimientos y acciones que caracterizaban a los enfermos mentales (o que en todo caso se les atribuía) como fuerza descomunal entre otros. La enfermedad mental así tornaba a ser de origen material. Nicholas Robinson, médico inglés, discípulo de Willis, lo expresó en forma clara en 1729: Every change of the mind indicates a change in the bodily organs.

La consecuencia inmediata fue la reincorporación de la locura entre las afecciones que podían ser tratadas por la medicina ya que subyacía a ella un desorden orgánico. El propio Robinson recomendaba frecuentes sangrías, ya que consideraba que dependía de una constitución corporal biliosa, cálida y sanguínea ${ }^{(8)}$, la cual requería dicho tratamiento. La interpretación somática y neurológica de la enfermedad mental recibió nuevas denominaciones no derivadas de las formas de presentación de la enfermedad, sino del rango social de quien la padece; así, la melancolía será denominada spleen cuando quien la padece pertenece a la clase superior, pero si quien presenta similar cuadro clínico es alguien de la clase trabajadora se le llamará "falta de espíritu" (lowness of spirit) ${ }^{(9)}$.

Los grandes sistemáticos de finales del siglo XVII como Herman Boerhaave y Von Haller difundieron la concepción neural de la enfermedad mental. Haller, el creador del termino "fisiología", estableció que la melancolía era el resultado de la evaporación de las partes más sutiles de la sangre y el consiguiente acúmulo y condensación de sus residuos térreos en el cuerpo, lo que causaba el letargo característico; el problema médico sería así el origen de esa condensación lo que dio lugar a las más diversas teorías. Erasmus Darwin, por ejemplo, relacionaba la hipocondría con la mala digestión. La concepción popular de la locura por la época también se tiñe de mecanicismo, la expresión popular "tener piedras en la cabeza" parece ser el origen de diversas pinturas sobre el tema artístico denominado Pierre de têtê o Pierre de folie (piedra de la locura) que retrata a un cirujano o charlatán extrayendo una piedra, la causa de su enfermedad, de la cabeza de un enfermo mental. Aun en su primitivismo subyace a dicha creencia popular una visión naturalista y mecanicista de la locura, desechando así, al menos parcialmente, la idea de posesiones demoniacas ${ }^{(10)}$. Estos cambios no implicaron que la locura se considerara un problema de salud pública, su carácter no contagioso, su limitada letalidad la alejaba de los problemas de salud pública $y$, en cambio, por la incapacidad de trabajar de quienes la padecían se asimiló paulatinamente al grupo de los pobres y enfermos que no pueden trabajar y que deben estar bajo la protección o cuidado del gobierno local.

El paradigma neurológico de la enfermedad mental cambia a partir de la difusión de las ideas de John Locke y el sensualismo de Condillac quienes frente al postulado cartesiano de las ideas innatas postularon que la mente es como una hoja en blanco (tabula rasa) en la cual se generan las ideas a partir de las sensaciones que provienen de los sentidos. William Cullen (1710-1790) desde Edimburgo, difundió esta interpretación en lo referido a las enfermedades mentales postulando que la locura (vesania) era una inusual y apresurada asociación de ideas que daba lugar a juicios equivocados sobre la realidad y, por 
consiguiente, a emociones desproporcionadas. Uno de sus discípulos, Thomas Arnold, elaboró sobre esta base una nueva taxonomía de la enfermedad mental, clasificándola en dos grandes grupos, aquellos que incluían delusiones, la "locura nocional" y los que presentaban alucinaciones, la "locura ideática"; ambas causadas por una errada relación entre las sensaciones y las ideas que se formaban a partir de ellas ${ }^{(11)}$.

En esa línea es que debe interpretarse la obra de figuras emblemáticas de la psiquiatría del siglo XVIII, como Vincenzo Chiarugi y Philippe Pinel, recordados por iniciar un trato humano a los enfermos mentales, liberándolos de cepos y cadenas, pero no se recuerda que este trato se deriva de una teoría subyacente, que en el caso de Chiarugi conlleva a una reinterpretación de la clasificación de la locura hecha por Cullen y que estaba en relación a los humores. Chiarugi mantiene la clasificación de William Cullen, (melancolía, manía y demencia), pero le atribuye un distinto significado a cada uno: La melancolia è una Pazzia parziale, e sempre limitata ad uno, o pochi oggetti relativi. La mania è una Pazzia universale con accompagnamento di audacia, e furore nelle operazioni Della volontà. L'amenza è una Pazzia universale, o quasi universale, con irregolarità d'azione nelle Potenze Intellettuali, e volontarie, ma naturalmente senza emozioni ${ }^{(12)}$.

Chiarugi incorpora el componente "psicológico" a la enfermedad mental, los estados corporales influenciarían la mente a través de la actividad de los sentidos y, en general, del sistema nervioso, lo que llamó el sensorium commune; por tanto, desechó que la enfermedad mental fuera hereditaria y se inclinó a considerarla adquirida. De allí deriva su principio terapéutico, basado en ejercer influencias positivas sobre los sentidos y el sistema nervioso. Estas medidas basadas en el ejemplo y superioridad moral no podían dejar de estar vinculadas a sus particulares convicciones morales y religiosas, lo cual se evidencia en sus historias clínicas en las que atribuye, por ejemplo, el origen de la melancolía al abuso de la masturbación.

Philippe Pinel también se inclina al aspecto psicológico, pero a partir de la imposibilidad de encontrar alteraciones morfológicas en las necropsias de enfermos mentales. A diferencia de Chiarugi que priorizaba como elemento terapéutico el "ejemplo", él enfatizó los aspectos emocionales en la terapéutica; añadió a la clasificación entonces en boga la manie sans délire, una forma de alteración mental sin deterioro de las funciones intelectuales. Se inicia en este periodo la era de los asilos en los cuales la abundancia de observaciones permite identificar y conocer mejor diversas enfermedades neurológicas con un compromiso de las facultades mentales. Tal es el caso de la descripción del petit mal por Esquirol y luego de la crisis de ausencia que precede a un ataque epiléptico por Calmell. Los asilos también incorporan entre sus internos numerosos pacientes afectados por la sífilis terciaria, identificada por Bayle en 1822.

La historia de los asilos es un tema amplio que excede los alcances del presente artículo; signados por una leyenda negra son múltiples las referencias a insanos enjaulados, confinados en cajas de madera o encadenados a celdas, pero sin duda representan el triunfo definitivo de la incorporación de la locura y los problemas de salud mental como un tema médico y, por ende de la Salud Pública. Lord Shaftesbury, responsable de una comisión de reforma de los asilos del parlamento inglés atribuía esta situación a los medicos: I could affirm that medical men who had not made the subject a special study, were as ignorant of mental disease as any who observed it for the first time ${ }^{(13)}$.

\section{LA PSIQUIATRÍA ACTUAL Y SUS DEBATES GENÉTICOS}

El titulo de este apartado no pretende hacer referencia a una determinación genética del origen de la enfermedad mental, sino a los debates que atraviesan la psiquiatría contemporánea y que podemos remontar en lo fundamental a la segunda mitad del siglo $X X$. Hasta 1950 la Psiquiatría y su historia era un ejemplo destacado de cómo una evolución disciplinaria había desterrado la barbarie y la ignorancia, y el tratamiento con torturas y confinamiento de los "lunáticos"; todo lo cual había sido paulatinamente desplazado por un tratamiento científico.

En esa aparente calma surgen posiciones como las de Szasz, psiquiatra norteamericano, quien en sus libros The myth of mental illness, 1961 y The manufacture of madness, 1971, niega la existencia de la enfermedad mental, la que sería un "mito" que han inventado los profesionales de la Psiquiatría alentados por una sociedad que así encuentra soluciones fáciles a los complejos problemas del ser humano, etiquetando como enfermos mentales a aquellos considerados como pestes sociales. Este punto de vista es más ampliamente conocido por otro de sus sostenedores, el filosofo Michel Foucault, historiador de las ideas, quien en Madness and civilization (1961) considera que la historia de la Psiquiatría no sería un recuento de los esfuerzos para combatir la enfermedad mental sino la historia de la derrota de la libertad por el control y del predominio del poder sobre el conocimiento. 
Naturalmente, se han presentado refutaciones, entre ellas la de Roth y Kroll (1981), quienes en The reality of mental illness (1986) refieren que la estabilidad de los signos de los síntomas psiquiátricos son la evidencia de que no responden a un constructo social sino a una entidad natural con una base orgánica ${ }^{(14)}$.

A comienzos del siglo $\mathrm{XXI}$, y con un evidente incremento de los problemas de salud mental, debates como el señalado nos señalan la necesidad de profundizar no solo en el tratamiento de la enfermedad mental y de su inclusión como problema prioritario de salud pública sino en encontrar las raíces de nuestro pensamiento sobre la locura y sus implicancias sociales.

\section{REFERENCIAS BIBLIOGRÁFICAS}

1. Householder F. Quem deus vult perdere dementat prius The Classical Weekly. 1936;29(21):165-7.

2. Hipocrates. Sobre la enfermedad sagrada. En: Tratados Hipocráticos Vol. I. Madrid: Ed Gredos; 1990. p. 399-400.

3. Faraone CA. Magical and medical approaches to the wandering womb in the ancient Greek world. Classical Antiquity. 2011;30(1):1-32.

4. Elgood $C$. The medicine of the prophet. Med Hist. 1962;6(2):146-53.

5. Dols MW. Insanity and its treatment in Islamic society. Med Hist. 1987;31(1):1-14
6. Bruce-Chwatt LJ. A medieval glorification of diseases and death. Med Hist. 1972;16(1):76-7.

7. de Rotterdam E. Elogio de la locura. Coloquios. Mexico: Ed Porrúa; 1990.

8. Robinson N. A new system of the spleen, vapours and hypocondriack melancholy. En: Ingram A (ed.) Patterns of madness in the eighteen century. London: Liverpool University Press; 1998. p 76.

9. Skultans V. English Madness. Ideas in insanity 1580-1890. London: Routledge; 1979.

10. Schupbach W. A new look at the cure of folly. Med Hist. 1978;22(3):267-81.

11. Arnold T. Observations on the nature, kinds, causes and prevention of insanity. Vol II $2^{\mathrm{a}}$ ed. London: Richard Phillips; 1806.

12. Chiarugi V. Chapitre IV: Divisione generale delle piazze. En: Della pazzia in genere, e in specie. Trattato medico analitico. Florence: Luigi Carlieri ed.; 1746. p. 46.

13. Hodgkinson RG. Provision for pauper lunatics 1834-1871. Med Hist. 1966;10(2):138-54.

14. Porter R. Madness: A brief History. London: Oxford University Press; 2002.

Correspondencia: Oswaldo Salaverry García

Dirección: Cápac Yupanqui 1400, Lima 11, Perú.

Teléfono: (511) 6176200 anexo 1609

Correo electrónico: oswaldosalaverry@gmail.com

\section{Consulte la versión electrónica de la Revista Peruana de Medicina Experimental y Salud Pública en}

\section{wWW.scopus.com}

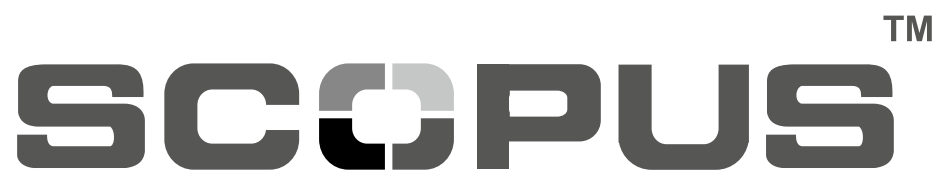

